\title{
A Study of Anycast Application for Efficiency Improvement of Multicast Trees
}

\author{
Kwang-Jae Lee', Won-Hyuck Choi ${ }^{2 *}$, and Jung-Sun Kim² \\ ${ }^{1}$ School of Electronics, Electronics and Multimedia, Seonam University, 702, Kwangchi-dong, \\ Namwon-city, Jeollabuk-do, 590-711, Korea \\ $\mathrm{kjlee}$ seonam.ac.kr \\ ${ }^{2}$ School of Electronics, Telecommunication and Computer Engineering, Hankuk Aviation \\ University, 200-1, Hwajeon-dong, Deokyang-gu, Koyang-city, Kyonggi-do, 412-791, Korea \\ rbooo@korea.com, jskim@mail.hangkong.ac.kr
}

\begin{abstract}
In this paper, we considered previously existing multicast routing algorism and protocol and especially put more attention on CBT routing protocol to analyze its strength and weakness. As a result, traffic was converged upon core router because of structural problem of CBT protocol and according to the converged traffic, there was congestion at the core link, thus it caused efficiency degrades of the whole routing. Therefore, we proposed a way of conversion to Anycast Routing method from the method of CBT multicast tree routing that was suitable for traffic decentralization even though there was high bandwidth depended on increment of traffic load. In order to support multimedia service that requires from small to large bandwidth and to consider multicast routing protocol to improve characteristic of multicast packet's delay, $\mathrm{CBT} /$ Anycast routing method can be proposed as an alternative plan for freedom of bandwidth in traffic.
\end{abstract}

Keywords: Internet applications, multicast routing, anycast routing, core base tree

\section{Introduction}

Multicast protocol classifies network users into specific groups and provides not only various but characterized services with communicating protocol to individuals, enterprises, and the government. It becomes a matter of concern and interest for internet communication.

The CBT (Core Base Tree) method, the representative protocol of the covalent tree, is one of methods to improve high-speed transmission of multicast packet and efficiency of communication by decreasing overhead from tree constitution's overlap. However, CBT (Core Base Tree) has several problems in structure and they work as its vulnerability (Core Base Tree) [3], [4].

The first problem of CBT is the phenomenon of transmitter's traffic concentration around Core Router. For instance, traffic density and surplus sign around Core router that are often seen in services like video, Telnet, Ftp, etc. Fig. 1 shows concentration problem in traffic and Fig. 2 is Poor Core phenomenon.

\footnotetext{
* The corresponding author will reply to any question and problem from this paper
} 
The core's ideal position in traffic reception is right in the middle that correspondent with the size of distance from group members.

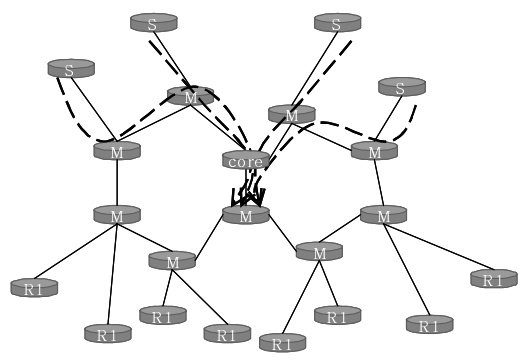

Fig. 1. Traffic concentration

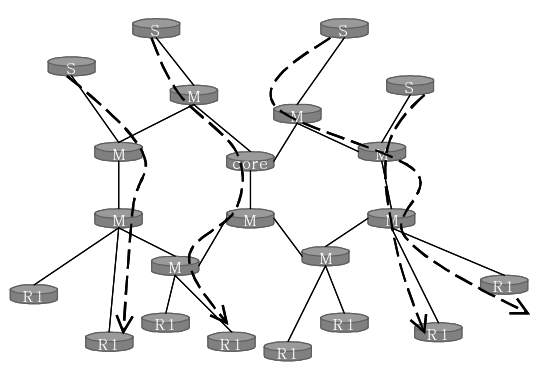

Fig. 2. Poor core placement

However, if the core is positioned in an isolated area from transmitter-recipient of packet and used independently, then it becomes impossible to have right choice and practice even though it does not require much the high bandwidth and the maintenance space of routing information. Therefore, ABT (Anycast Based Tree) is proposed in the paper.

ABT does not limit core in specific position within network but let it actively be located so that the previously mentioned problems of CBT can be solved. The specific resolution is to use AIMD (Addictive Increase Multiple Decrease) algorism. The controlled transmission rate of traffic enables traffic that is concentrated in core router, to maintain average transmission rate and leads traffic to poor core so it helps to improve excess use in whole system and performs multicast service in high speed [6], [7].

\section{ABT}

The main characteristic of the suggested ABT is its treatment of multicast packet in a formation like CBT without having core router. In this process, however, it requires control mechanism that moves traffic to core in the other side when traffic gets concentrated in core more than threshold.

In multicast routing, the time for traffic to pass the core is called Core round trip time (crtt), and crtt becomes reset time for a table in a transmitter and a control parameter.

For increase factor of packet, the transmission time increment of core can be shown as $a / c r t t$, and if there is increase in transmitter, the formula is like below:

$$
R_{\text {in }}=R_{\text {now }}+\frac{a}{c r t t} .
$$

where, $R_{\text {in }}$ is transmit packet and $R_{\text {now }}$ is amount of packet in the present core. If there is decrease of packet to core, the formula becomes like below:

$$
R_{\text {in }}=\frac{R_{\text {now }}}{b} .
$$


where, $b$ is factor for decrease. The increase of transmitted packet and the average transmitted rate based on decrease can be calculated at the core from $a$ and $b$. Also, the transmission rate is calculated according to the size of packet from recipient and the minimum and maximum rate for transmission can be calculated with increase of recipient as follows:

$$
\begin{aligned}
R_{\max } & =R_{i n} \cdot \frac{a}{c r t t} \cdot n . \\
R_{\min } & =\frac{R_{\max }}{b} .
\end{aligned}
$$

$R_{\min }$ is minimum rate for transmission of core, $R_{\max }$ is maximum rate for transmission of core, and transmission time increase is $n$. The below formula is for the average rate for transmission by using minimum and maximum rates for transmission of core:

$$
R_{\text {ave }}=\frac{R_{\max }+R_{\min }}{2}=\frac{a}{2} \cdot \frac{b+1}{b-1} \cdot \frac{n}{c r t t} .
$$

According to Eqn 5, Poor Core phenomenon that becomes the minimum rate for transmission of core and congestion around core that occurs it becomes the maximum rate of transmission can be controlled with the average rate of transmission.

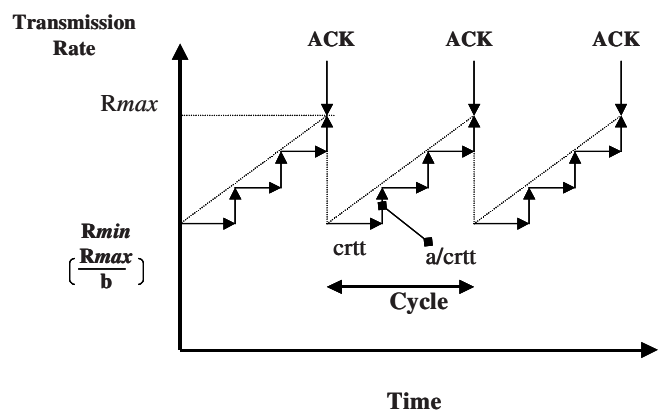

Fig. 3. The rate of transmission based on increase in transmission

Fig. 3 shows retransmission of ACK at the end of each cycle after the multicast packet ( $\mathrm{n}, \mathrm{crtt}$, Rave) completed transmission. The formula for the average rate for transmission in core router is like below. At this time, loss of packet through retransmission of ACK is $p$.

$$
\text { Trans }=\frac{1}{c r t t} \sqrt{\frac{a}{2} \cdot \frac{b+1}{b-1} \cdot \frac{1}{p} .}
$$

\section{Simulation and Discussion}

To the simulation model, each CBT routing protocol is applied and the numbers of multicast groups and transmitters are varied. Then the packet process condition of Core router is measured based on the sized of multicast data packet. Fig. 4 and Fig. 5 show the result of the simulation. The reason for this is that there is formation of 
initialization of multicast tree and is frequent Join and Leave of group, thus interval for packet's arrival becomes shorter and relatively increase in load of packet occurs as a result. Fig. 4 shows packet transmission delay of CBT and Fig. 5 shows cuing delay of core as the system is executed as Anycast routing protocol in CBT routing protocol.

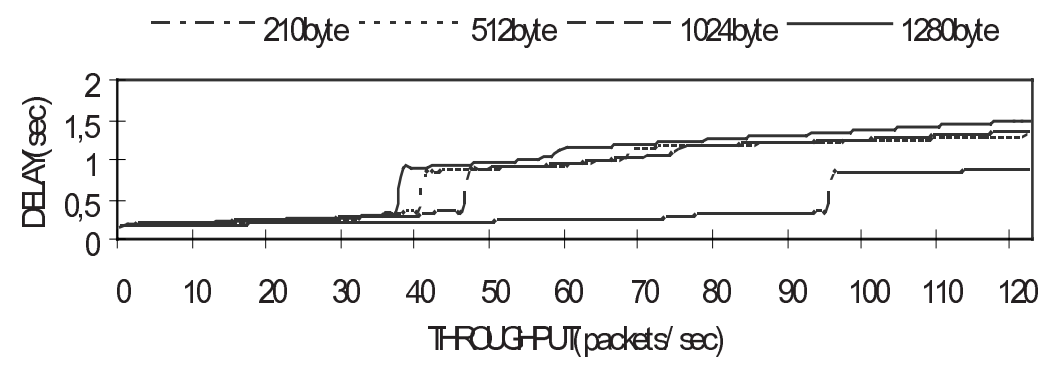

Fig. 4. Packet transmission delay of CBT

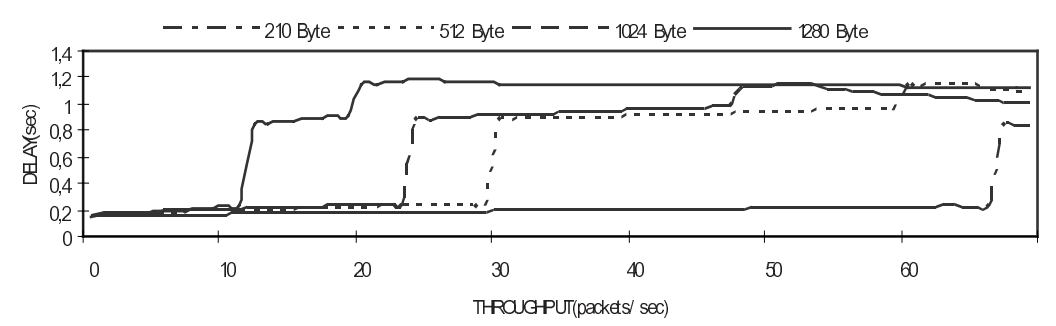

Fig. 5. Packet transmission delay of CBT/Anycast core

In this paper, change of routing methods from CBT shared tree routing method that is stable in relatively low bandwidth to Anycast routing method that is appropriate for traffic congestion even in high bandwidth depends on load of traffic. In the study, characteristics of delay according to the size change of multicast data packet when the system is changed from CBT to Anycast, were observed and evaluated.

\section{References}

1. Parsa, M., Garcia-Luna-Aceves, J. J.: A protocol for scalable loop-tree multicast routing. IEEE J. Select. Areas Commun. 15 (1997) 316-331

2. Jia, X., Wang, L.: A Group Multicast Routing Algorithm by using Multiple Minimum Steiner Trees. Computer Communications (1997) 750 -758

3. Ballardie, A.: Core Based Trees (CBT) Multicast Routing Architecture. RFC2201 (1997)

4. Ballardie, A.: Core Based Trees (CBT Version 2) Multicast Routing Protocol Specification. RFC2189 (1997)

5. Moy, J.: Multicast Extensions to OSPF. IETF RFC1584 (1994)

6. Ettikan, K.: An Analysis of Anycast Architecture And Transport Layer Problems. Asia Pacific Regional Internet Conference on Operational Technologies (2001)

7. Lin, J., Paul, S.: RMTP: A Reliable Multicast Transport Protocol. IEEE INFOCOM96 (1996) 